\title{
JNVESTGAATONN
}

\section{Composición química de aceites de las variedades Arbequina y Empeltre cultivadas en regadío}

\author{
Por Mª Soledad Gracia ${ }^{1^{*}}$, Antonio Royo² y Mónica Guillén ${ }^{2}$ \\ ${ }^{1}$ Laboratorio Agroalimentario. Gobierno de Aragón. Avda. Montañana, 1005. 50071 Zaragoza \\ ${ }^{2}$ Unidad de Suelos y Riegos (Unidad Asociada al CSIC). Centro de Investigación \\ y Tecnología Agroalimentaria de Aragón (CITA). Avda. Montañana 930, 50059 Zaragoza \\ (*autor para la correspondencia: msgracia @ aragon.es)
}

\section{RESUMEN}

Composición química de aceites de las variedades Arbequina y Empeltre cultivadas en regadío.

Este trabajo analiza la composición de aceites de árboles jóvenes de las variedades Arbequina y Empeltre cultivados en regadío en una misma finca. Durante tres campañas se estudiaron los parámetros de calidad, la composición en ácidos grasos, los esteroles, las ceras y los alcoholes alifáticos. Aunque la calidad de los aceites de las dos variedades fue la de virgen extra se encontraron diferencias varietales. El índice de peróxidos y los polifenoles totales tuvieron valores muy superiores en la variedad Empeltre, sin embargo la estabilidad fue mayor en Arbequina. El total de ácidos monoinsaturados fue mayor en Arbequina y el de poliinsaturados en Empeltre. Arbequina contenía más esteárico, oleico, araquídico y behénico que Empeltre, en tanto que en esta variedad era mayor en palmítico, margaroleico, linoleico, linolénico, gadoleico y lignocérico. La composición esterólica fue también diferente en las dos variedades, con mayores contenidos de 24-metilencolesterol, campesterol y, especialmente en $\Delta-5$ Avenasterol en Arbequina, y de campestanol, $\Delta$-7 Estigmastenol, $\Delta-7$ Avenasterol en Empeltre. Tanto el contenido en ceras como el de alcoholes alifáticos fueron mayores en Arbequina.

PALABRAS CLAVE: Ácidos grasos - Alcoholes alifáticos - Ceras - Esteroles - Parámetros de Calidad.

\section{SUMMARY}

Chemical composition of olive oils from Arbequina and Empeltre varieties grown in irrigation.

This study examines the composition of oils extracted from young olive trees of the Arbequina and Empeltre varieties grown with irrigation in the same farm throughout a period of three seasons. Quality parameters, Fatty acid composition, sterols, waxes and aliphatic alcohols were analyzed. Although the quality of oils of both varieties was that of extra virgin, varietal differences were found. The peroxide value and the content of total polyphenols were much higher in Empeltre, whereas stability was higher in Arbequina. The monounsaturated acids were higher in Arbequina containing more stearic, oleic, arachidic and behenic acids than Empeltre, while this variety surpassed Arbequina in palmitic, margaroleic, linoleic, linolenic, gadoleic and lignoceric acids. The sterol composition was also different in the two varieties with higher contents of 24-methylencholesterol, campesterol and, especially, $\Delta-5$ avenasterol in Arbequina, and campestanol, $\Delta-7$ stigmastenol, $\Delta-7$ Avenasterol in Empeltre. The contents of both waxes and aliphatic alcohols were higher in Arbequina.

KEY-WORDS: Aliphatic alcohols - Fatty acids - Quality parameters - Sterols - Waxes.

\section{INTRODUCCIÓN}

España es el principal país productor de aceite de oliva con alrededor de $1.000 .000 \mathrm{Tm}$ de aceite de oliva virgen en 2006 (MAPYA, 2007). El olivar en España es el segundo cultivo en extensión, después de los cereales, ocupando cerca de 2.500 .000 ha, en el año 2006 de las que unas 400.000 estaban en regadío. A esa superficie habría que sumar los 160.000 árboles que se encuentran diseminados. En Aragón es también un cultivo importante con más de 46.000 ha.

En general, el olivar ha sido un cultivo tradicional con problemas muy diversos (cultivados en suelos pobres y erosionados, en terrenos con pendiente, con frecuentes problemas de plagas, de vecería, etc.). El olivar ha tenido importancia histórica como generador de trabajo en otoño y comienzos de invierno cuando la demanda de trabajo en el campo es escasa, generando del orden de 70-90 horas de trabajo anual por ha, de las cuales de 50 a 65 corresponden a las labores de recolección. La olivicultura moderna, con nuevas variedades, densidades de plantación intensivas, instalación de sistemas de riego localizado, tratamientos fitosanitarios y culturales, mecanización de la cosecha, etc. permitirán conseguir mayores rendimientos, una disminución de los costes de producción y una mejora de la calidad del aceite.

El rendimiento y la calidad del aceite de oliva son producto de la integración de diversos factores tanto agronómicos como industriales. Los primeros 
afectan directamente a la oliva y de ahí a la calidad del aceite; algunos son modificables por el agricultor, y se refieren a las prácticas culturales. Otros difícilmente se pueden modificar como la variedad y el medio. Sin embargo, las diferencias de composición del aceite para una misma variedad pueden llegar a ser importantes dependiendo del medio agrológico en el que crecen los olivos. La comparación de la calidad de aceites de dos variedades debería hacerse sobre muestras procedentes de árboles cultivados de forma idéntica y en el mismo medio.

Empeltre es la variedad más extendida en el Valle del Ebro e Islas Baleares, cultivándose una superficie de unas 80.000 ha de las que 48.000 lo son en la comunidad de Aragón (Tous et al., 2004). El Reglamento de la Denominación de Origen "Aceite del Bajo Aragón" establece que las variedades de aceituna aptas para la elaboración de aceites protegidos por dicha denominación son Empeltre, que participará en una proporción mínima del $80 \%$, y Arbequina y Royal que no podrán superar el $20 \%$. Sin embargo, añade que el Consejo Regulador podrá proponer nuevas variedades, siempre que se conserven las características de los aceites de la Denominación de Origen. Arbequina es la variedad que más se está extendiendo en las nuevas plantaciones de regadío, especialmente en forma intensiva y superintensiva, por su menor desarrollo y precocidad en la entrada en producción.

En este trabajo se comparan los aceites de las dos variedades Arbequina y Empeltre cultivados en regadío en la misma finca.

\section{MATERIALES Y MÉTODOS}

\subsection{Material vegetal}

Las olivas y sus aceites correspondientes se obtuvieron de los árboles del tratamiento control de dos ensayos implantados en la finca del Centro de Investigación y Tecnología Agroalimentaria de Aragón en Montañana (Zaragoza). En uno de los ensayos se trataba de ver la respuesta del olivo al riego deficitario, en tanto que en el otro se estudió la influencia de la salinidad del agua de riego sobre la producción y calidad del aceite. Ambos ensayos estaban en una misma parcela separados entre sí unos $50 \mathrm{~m}$. El marco de plantación fue de $5,5 \mathrm{~m}$ entre filas y de $2,5 \mathrm{~m}$ entre árboles. En el ensayo de riego había tres repeticiones de cada tratamiento con tres árboles por repetición y variedad. En el ensayo de salinidad había dos repeticiones por tratamiento con cuatro árboles por variedad.

En este estudio se han utilizado los árboles del ensayo de riego que recibieron el $100 \%$ de la evapotranspiración del olivo (ETc), y los del tratamiento control, del ensayo de salinidad, regados con agua de la acequia Urdana con la que se regaron ambos ensayos.
La ETc se calculó como el producto de la evapotranspiración de referencia $\left(\mathrm{ET}_{0}\right)$ calculada mediante el método Penman-Monteith con los datos meteorológicos obtenidos en la propia finca experimental y los coeficientes de cultivo (Kc) obtenidos experimentalmente en zonas productoras de Andalucía (García-Fernández y Berengena, 1993) y de coeficiente reductor $(\mathrm{Kr})$ en función de la superficie sombreada por el árbol (Fereres et al., 1981).

El agua de riego se aplicó con un sistema de riego localizado de alta frecuencia. Ambos cv. Arbequina y Empeltre se regaron simultáneamente y con la misma frecuencia.

Aunque los árboles de la variedad Arbequina tuvieron cosecha ya en 2003, el estudio se centra en los años 2004, 2006 y 2007 en los que ambas variedades tuvieron producción. En el año 2005 no hubo cosecha por las heladas de febrero y marzo.

La recolección se hizo a mano, individualmente en cada árbol, empezando por los árboles de Empeltre cuyas olivas tenían un estado de maduración más avanzado que las de Arbequina. El valor medio del índice de madurez en las muestras de olivas de Empeltre fue de 4,1 en 2004, 6,3 en 2006 y 6,1 en 2007, en tanto que en Arbequina los valores fueron respectivamente 2,7, 4,3 y 5,5.

En el mismo campo se pesaba la cosecha de cada árbol y, si era necesario, se juntaban posteriormente las olivas de árboles de la misma repetición, para alcanzar la cantidad necesaria para la extracción de aceite. Al día siguiente de su recolección las olivas se pasaban al laboratorio donde eran procesadas inmediatamente. Se han analizado un total de 30 muestras, 17 de Arbequina y 13 de Empeltre, correspondiendo 9 muestras a 2004, 13 a 2006 y 8 a 2007.

\subsection{Extracción de aceite}

Aproximadamente un kilogramo de olivas de cada muestra se molturó con un molino de martillos, dotado de una criba de $5 \mathrm{~mm}$. De la pasta obtenida se tomó una muestra en la que se midieron simultáneamente humedad y grasa, utilizando un Olivescan de la marca FOSS, Type 78800. Es un método rápido indirecto NIR, basado en la tecnología NIT (Near Infrared Transmítance, Transmitancia en el Infrarrojo Cercano). El aparato tiene las calibraciones necesarias para dar los contenidos de \% de Grasa sobre materia natural, \% Grasa sobre materia seca y \% de Humedad.

Una cantidad de unos $750 \mathrm{~g}$ de la pasta se utilizó para la extracción del aceite por el sistema Abencor. Las condiciones de trabajo fueron: Temperatura del agua de la batidora, $28^{\circ} \mathrm{C}$, tiempo de batido total: $50 \mathrm{~min}$., con un minuto de centrifugación y un minuto más después de la adición de agua.

Después de decantado el aceite obtenido, se filtró con filtro de celulosa y se guardó en frascos de 
color topacio, en la oscuridad, a temperatura por debajo de $0{ }^{\circ} \mathrm{C}$ hasta su análisis.

\subsection{Análisis de aceites}

Se ha seguido la metodología descrita en el Reglamento n- 2568/91 de la CEE de 11 de julio de 1991, para la determinación del grado de acidez (\% de ácido oleico), del índice de peróxidos (miliequivalentes de oxígeno activo por kilo de grasa, meq $\mathrm{O}_{2} / \mathrm{kg}$ ), y de las lecturas en el ultravioleta: $\mathrm{K}_{270}, \mathrm{~K}_{232}$ calculadas a partir de la absorción a 270 y $232 \mathrm{~nm}$.

Para la determinación del contenido de polifenoles totales, se siguió el método descrito por Vázquez et al. (1973), cuantificándolos a partir de un complejo coloreado formado por el reactivo de Folin-Ciocalteau en un espectrofotómetro UV/VIS Hitachi U2000 a 725nm de longitud de onda, y expresando el resultado en $\mathrm{mg}$ de ácido caféico $/ \mathrm{kg}$ de aceite.

La estabilidad oxidativa, que se expresa como tiempo de inducción en horas, se midió con un aparato Rancimat 679 Metrohm usando una muestra de $2,5 \mathrm{~g}$ de aceite, a $120{ }^{\circ} \mathrm{C}$ y $20 \mathrm{l} / \mathrm{h}$ (Gutiérrez, 1989).

La composición de ácidos grasos se determinó siguiendo asimismo el Reglamento no 2568/91 de la CEE de 11 de julio de 1991 y posterior modificación Reglamento oㅜ 796/2002 de 6 de mayo de 2002; los esteres metílicos se prepararon a partir de $0,1 \mathrm{~g}$ de aceite disuelto en $2 \mathrm{ml}$ de hexano con $0,4 \mathrm{ml}$ de potasa metanólica $2 \mathrm{~N}$ y analizados por cromatografía de gases con un detector FID. Se utilizó una columna de sílice fundida CP-SIL88 de 50 $\mathrm{m}$. de longitud, $0,25 \mathrm{~mm}$ de diámetro interior y 0,2 $\mu \mathrm{m}$ de espesor; como gas portador se utilizó helio.

En el análisis de la composición esterólica, del contenido en eritrodiol y uvaol y el de los alcoholes alifaticos, se ha seguido la metodología descrita en el Reglamento $n^{\circ}$ 2568/91 de la CEE de 11 de julio de 1991, a partir del insaponificable, por separación de la fracción correspondiente, mediante cromatografía de capa fina, silanización y posterior análisis por cromatografía de gases, con detector FID, y columna SPB-5 de 30m de longitud, de 0,25 de diámetro interior, y 0,25 de espesor de $5 \%$ difenil-95\% dimetilsiloxano.

El contenido en ceras se obtuvo según el Anexo IV del Reglamento CEE 183/93 del 16 de febrero de 1993 por cromatografía de gases del aceite fraccionado mediante cromatografía en columna de gel de sílice hidratado. El detector FID, y columna capilar RTX-5, de $15 \mathrm{~m}$ de longitud, $0,32 \mathrm{~mm}$ de diámetro interior, con un recubrimiento interno de $5 \%$ de difenil-95\% dimetil polixilosano, de $0,1 \mu \mathrm{m}$ de espesor.

\subsection{Análisis estadístico}

Para los análisis de varianza se ha utilizado el procedimiento GLM del programa SAS versión 9.1. La comparación de medias se hizo con el test de Duncan. En el análisis cluster se ha utilizado el método de Ward y la distancia Euclídea

\section{RESULTADOS Y DISCUSIÓN}

\subsection{Olivas}

En la Tabla 1 se presentan los valores medios, la desviación estándar y los valores mínimo y máximo de la humedad, el contenido graso sobre materia natural y seca, el rendimiento en el Abencor y la extractabilidad para las dos variedades Arbequina $(A)$ y Empeltre $(E)$ analizadas con todos los datos.

Solo para la humedad se encontraron diferencias significativas entre variedades cuando se analizaron conjuntamente las muestras de todos los años, teniendo las olivas de Empeltre más humedad (53\%) que Arbequina (50\%), debido a su recolección más temprana. Pero analizando cada año por separado solo en 2004 fue significativamente inferior la humedad de Arbequina $(48 \%)$ a la de Empeltre (58\%).

Tabla 1

Valores medios de humedad, grasa sobre materia fresca y sobre materia seca, rendimiento industrial (Abencor) y extractabilidad en las olivas de las variedades Arbequina $(A)$ y Empeltre $(E)^{a}$

\begin{tabular}{lccc}
\hline \multicolumn{1}{c}{ Parámetro } & Variedad & Media \pm Desvest & Rango \\
\hline \multirow{2}{*}{ Humedad } & $\mathrm{A}$ & $50,1 \pm 5,9 \mathrm{~b}^{*}$ & $40,8-60,8$ \\
& $\mathrm{E}$ & $53,2 \pm 7,1 \mathrm{a}$ & $42,4-50,5$ \\
Grasa /materia seca & $\mathrm{A}$ & $47,7 \pm 3,3 \mathrm{a}$ & $43,5-52,9$ \\
& $\mathrm{E}$ & $46,8 \pm 2,0 \mathrm{a}$ & $43,7-50,5$ \\
Grasa /materia fresca & $\mathrm{A}$ & $23,9 \pm 3,8 \mathrm{a}$ & $17,9-31,3$ \\
& $\mathrm{E}$ & $22,0 \pm 4,2 \mathrm{a}$ & $16,4-29,1$ \\
Abencor & $\mathrm{A}$ & $16,2 \pm 3,8 \mathrm{a}$ & $10,5-22,8$ \\
& $\mathrm{E}$ & $15,8 \pm 4,6 \mathrm{a}$ & $9,9-23,3$ \\
Extractabilidad & $\mathrm{A}$ & $67,2 \pm 5,9 \mathrm{a}$ & $55,8-74,8$ \\
& $\mathrm{E}$ & $70,5 \pm 7,9 \mathrm{a}$ & $56,8-80,2$ \\
\hline
\end{tabular}

\footnotetext{
${ }^{a}$ Se presentan la desviación estándar y el intervalo de variación.

* Para cada carácter analizado medias seguidas de la misma letra no son significativamente diferentes $(P<0,05)$.
} 
En todos los caracteres hay una influencia clara del año, siendo el año 2007 el que presenta valores significativamente mayores de grasa, rendimiento Abencor y extractabilidad.

\section{2. Caracteres de calidad del aceite}

En la Tabla 2 aparecen los valores medios con la desviación estándar y los valores extremos para la acidez (\% oleico), el índice de peróxidos, la absorbencia en el ultravioleta $(\mathrm{K})$ a las longitudes de onda de 270 y $232 \mathrm{~nm}$, los polifenoles y la estabilidad. Excepto para el K270 y la estabilidad, se han encontrado diferencias significativas tanto entre las dos variedades, como entre los diferentes años.

Los aceites obtenidos de los árboles de Arbequina y Empeltre fueron de baja acidez, con valor medio ligeramente superior en Arbequina, que fue significativamente mayor que la media de Empeltre.

Donde las dos variedades se diferencian notablemente es en el índice de peróxidos, ya que el vaIor medio en Empeltre triplica al de Arbequina. Se ha visto una influencia notable del año, resultando el 2007 diferente y mayor que los años 2004 y 2006. Esta diferencia entre los años es también manifiesta en Arbequina, en la que los valores medios de los peróxidos en los tres años analizados son diferentes entre sí. Sin embargo, no hay diferencias entre años en Empeltre, aunque el valor del 2007 fuera superior al de los otros dos años. Comparando estos valores con los citados en otros trabajos, Gracia (2001) en aceites de Empeltre de diversas procedencias, obtuvo valores menores y con escasa variabilidad entre años. Además en un estudio sobre el momento óptimo de recolección en esa variedad, pero con árboles adultos, los aceites obtenidos tuvieron valores del índice de peróxidos que variaban entre 2,5 y 4,5 meq de $\mathrm{O}_{2} / \mathrm{kg}$ de aceite (Gracia et al., 2005). En Arbequina, los valores aquí obtenidos son similares a los presentados por
Gracia (1996, 2005) y por Faci et al (2002), aunque Cert et al. (1996), Torres y Maestri (2006) y Allouche et al (2007) obtuvieron valores más altos. Salvo dos muestras de Empeltre de 2007, el resto de aceites tuvieron un índice de peróxidos por debajo del límite máximo admisible de 20 meq de $\mathrm{O}_{2} / \mathrm{kg}$ de aceite.

La absorbencia en el ultravioleta a la longitud de onda de $270 \mathrm{~nm}$ no dio resultados diferentes para las dos variedades, ni cuando se analizaron el conjunto de años ni cuando los datos se analizaron para cada año individualmente. Todos los aceites tuvieron un $\mathrm{K}_{270}$ inferior a 0,22 , límite máximo que marca el Reglamento para los aceites virgen extra.

El valor medio del $\mathrm{K}_{232}$ de Empeltre resultó más elevado que el de Arbequina, tanto en el análisis anual individual como en su conjunto, siendo estas diferencias significativas $(P<0,05)$. Por otra parte, el valor medio del año 2004 fue significativamente inferior al de los otros dos años. Los valores medios de las dos variedades no son diferentes de los obtenidos en otros trabajos, en los que el valor del $\mathrm{K}_{232}$ es menor en Arbequina que en Empeltre (Cert et al. 1996, Faci et al. 2002, Gracia 1996 y 2005, Torres y Maestri, 2006 y Allouche et al. 2007).

El contenido en polifenoles es otro carácter que marca grandes diferencias entre Arbequina y Empeltre, ya que esta variedad tenía un valor medio de 167, en tanto que en Arbequina era de 93. La cantidad de polifenoles fue significativamente distinta en los tres años. Esta diferencia en el contenido de polifenoles entre las dos variedades fue mucho mayor en 2004 y algo menor en 2006. El efecto del año sobre el contenido de polifenoles ha sido destacado por Mailer et al (2005).

La diferencia entre los contenidos en polifenoles de las dos variedades se manifiesta también en otros trabajos. Así, para árboles adultos de Empeltre, Gracia (2001) da valores de 160 a 346, depen-

Tabla 2

Valores medios con desviación estándar y mínimo y máximo de los caracteres de calidad de aceites de Arbequina (A) y Empeltre (E)

\begin{tabular}{lccc}
\hline \multicolumn{1}{c}{ Parámetro } & Variedad & Media \pm Desvest. & Rango \\
\hline \multirow{2}{*}{ Acidez } & $\mathrm{A}$ & $0,14 \pm 0,05 \mathrm{a}^{*}$ & $0,08-0,27$ \\
\multirow{2}{*}{ Índice de peróxidos } & $\mathrm{E}$ & $0,11 \pm 0,03 \mathrm{~b}$ & $0,07-0,16$ \\
& $\mathrm{~A}$ & $5,1 \pm 2,0 \mathrm{~b}$ & $2,0-9,0$ \\
K270 & $\mathrm{E}$ & $16,8 \pm 9,0 \mathrm{a}$ & $8,4-37,0$ \\
& $\mathrm{~A}$ & $0,09 \pm 0,01 \mathrm{a}$ & $0,06-0,12$ \\
K232 & $\mathrm{E}$ & $0,09 \pm 0,02 \mathrm{a}$ & $0,06-0,14$ \\
& $\mathrm{~A}$ & $1,44 \pm 0,12 \mathrm{~b}$ & $1,22-1,61$ \\
Polifenoles & $\mathrm{E}$ & $1,69 \pm 0,18 \mathrm{a}$ & $1,46-2,08$ \\
& $\mathrm{~A}$ & $93,3 \pm 41,6 \mathrm{~b}$ & $36-188$ \\
Estabilidad & $\mathrm{E}$ & $166,7 \pm 93,7 \mathrm{a}$ & $64-399$ \\
& $\mathrm{~A}$ & $7,5 \pm 1,9 \mathrm{a}$ & $5,0-11,2$ \\
& $\mathrm{E}$ & $6,9 \pm 1,6 \mathrm{a}$ & $4,9-9,7$ \\
\hline
\end{tabular}

* Para cada carácter analizado medias seguidas de la misma letra no son significativamente diferentes $(P<0,05)$. 
diendo de los años, en tanto que Tous et al (2004) señalan un valor medio de $299 \pm 156$ y Gracia et al. (2005) dan valores entre 141 y 344, dependiendo del momento de la recolección. Por el contrario, en Arbequina las cifras de polifenoles han variado entre 82 ppm de ácido caféico (Allouche, 2007) y 347 (Tous et al, 1997). Faci et al. (2002) en un ensayo de dosis de riego en árboles adultos de Arbequina dan valores de polifenoles que van desde $348 \mathrm{ppm}$ en el tratamiento regado al $100 \%$ de sus necesidades hasta 681 en los árboles que no tuvieron riego.

La estabilidad, a diferencia del contenido en polifenoles no resultó diferente en las dos variedades, ni anualmente ni en el conjunto de años. Este resultado parecería contradictorio con el de los polifenoles dada la relación que existe entre este parámetro y la estabilidad (Salas et al. 1997, Salvador et al., 2001). El coeficiente de correlación entre el contenido en polifenoles y la estabilidad para el conjunto de las 31 muestras analizadas fue de 0,58 . Sin embargo, si separamos los datos de las dos variedades como se muestra en la Figura 1 se observa que los ajustes son mejores (0,60 en Arbequina y 0,79 en Empeltre). Se observa asimismo, que la pendiente de la regresión de la estabilidad en función del contenido en polifenoles es mayor en Arbequina. Es decir, para un mismo contenido en polifenoles, la estabilidad es menor en la variedad Empeltre. Esto es posible por el mayor contenido en peróxidos de esta variedad y por la composición en ácidos grasos, como se indica a continuación.

\section{3. Ácidos grasos}

Aunque los porcentajes de cada ácido graso son muy similares en las dos variedades con diferencias en valor absoluto muy pequeñas, solamente los ácidos palmitoleico (C16:1) y margárico
(C17:0) han resultado no diferentes; para el resto de ácidos grasos hay diferencias significativas entre las variedades, como se muestra en la Tabla 3. Así, Arbequina contiene más esteárico (C18:0), oleico (C18:1), araquídico (C20:0) y behénico (C22:0) que Empeltre, en tanto que esta variedad supera a Arbequina en palmítico (C16:0), margaroleico (C17:1), linoleico (C18:2), linolénico (C18:3), gadoleico (C20:1) y lignocérico (C24:0). Las mayores diferencias entre variedades fueron en $\mathrm{C} 18: 3$ (30\%), C18:0 (21\%), C20:0 (20\%) y C18:2 (17\%).

En la Tabla 4 se presentan los valores medios y extremos del total de ácidos saturados insaturados, mono y poliinsaturados, las relaciones insaturados/saturados, monoinsaturados/polinsaturados y oleico/linoleico, así como la susceptibilidad oxidativa (Cert et al. 1996). No hay diferencias entre variedades en el contenido total de ácidos saturados, ni en el de insaturados, así como en la relación entre ellos. Sí se diferencian en los monoinsaturados que son mayores en Arbequina y en el de poliinsaturados que, por el contrario, son más abundantes en Empeltre. La relación entre monoinsaturados y poliinsaturados es también significativamente mayor en Arbequina.

Diversos trabajos (Aparicio et al. 1999; Poiana y Mincione, 2004) han mostrado la importancia de la relación oleico/linoleico en la calidad nutricional de los aceites y en su estabilidad oxidativa. Los aceites de la variedad Arbequina de este trabajo tenían un valor medio de la relación oleico/linoleico de 6,2 $\pm 1,3$, mayor estadísticamente que el valor en Empeltre que fue de 4,8 $\pm 0,5$. Considerando la relación en cada uno de los años, siempre el valor absoluto en Arbequina fue mayor que en Empeltre, pero solo en 2007 la diferencia llegó a ser significativa.

También son diferentes las variedades Arbequina y Empeltre por la susceptibilidad oxidativa, mayor estadísticamente en Empeltre. Esta diferencia

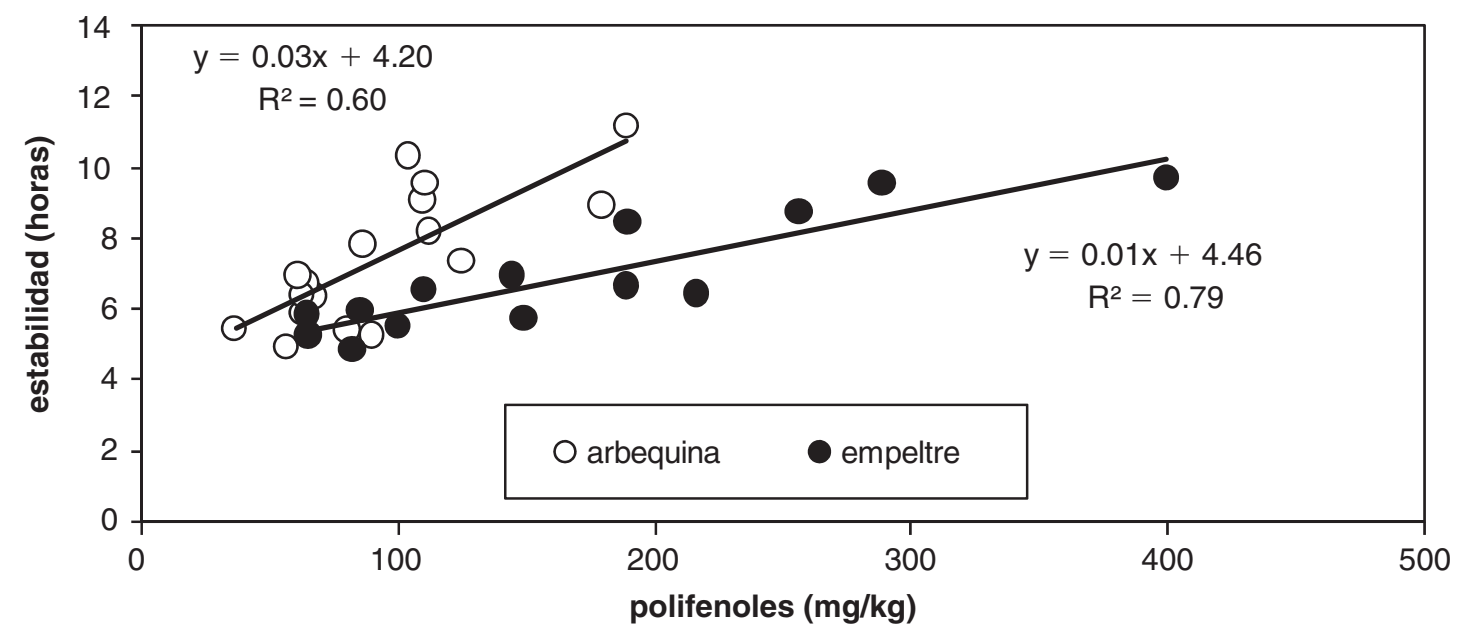

Figura 1

Relación existente entre el contenido total de polifenoles y la estabilidad en aceites de Arbequina y Empeltre. 
Tabla 3

Valores medios con desviación estándar y rango de variación de los ácidos grasos de los aceites de Arbequina (A) y Empeltre (E)

\begin{tabular}{lccc}
\hline Parámetro & Variedad & Media \pm Desvest. & Rango \\
\hline Palmítico & $\mathrm{A}$ & $14,1 \pm 1,1 \mathrm{a}^{*}$ & $12,1-15,5$ \\
C16:0 & $\mathrm{E}$ & $14,6 \pm 0,7 \mathrm{a}$ & $13,6-15,9$ \\
Palmitoleico & $\mathrm{A}$ & $1,77 \pm 0,57 \mathrm{a}$ & $0,97-2,73$ \\
C16:1 & $\mathrm{E}$ & $1,54 \pm 0,22 \mathrm{a}$ & $1,13-1,87$ \\
Margárico & $\mathrm{A}$ & $0,11 \pm 0,03 \mathrm{a}$ & $0,09-0,18$ \\
C17:0 & $\mathrm{E}$ & $0,11 \pm 0,01 \mathrm{a}$ & $0,09-0,13$ \\
Margaroleico & $\mathrm{A}$ & $0,26 \pm 0,04 \mathrm{~b}$ & $0,20-0,33$ \\
C17:1 & $\mathrm{E}$ & $0,30 \pm 0,02 \mathrm{a}$ & $0,28-0,33$ \\
Esteárico & $\mathrm{A}$ & $1,74 \pm 0,18 \mathrm{a}$ & $1,51-2,13$ \\
C18:0 & $\mathrm{E}$ & $1,46 \pm 0,09 \mathrm{~b}$ & $1,36-1,69$ \\
Oleico & $\mathrm{A}$ & $68,9 \pm 3,3 \mathrm{a}$ & $64,4-74,4$ \\
C18:1 & $\mathrm{E}$ & $66,3 \pm 1,6 \mathrm{~b}$ & $63,7-68,4$ \\
Linoleico & $\mathrm{A}$ & $11,6 \pm 1,8 \mathrm{~b}$ & $8,4-14,1$ \\
C18:2 & $\mathrm{E}$ & $13,9 \pm 1,2 \mathrm{a}$ & $12,4-16,2$ \\
Linolénico & $\mathrm{A}$ & $0,61 \pm 0,07 \mathrm{~b}$ & $0,48-0,71$ \\
C18:3 & $\mathrm{E}$ & $0,86 \pm 0,11 \mathrm{a}$ & $0,67-1,04$ \\
Araquídico & $\mathrm{A}$ & $0,37 \pm 0,02 \mathrm{a}$ & $0,35-0,42$ \\
C20:0 & $\mathrm{E}$ & $0,31 \pm 0,02 \mathrm{~b}$ & $0,27-0,35$ \\
Gadoleico & $\mathrm{A}$ & $0,31 \pm 0,02 \mathrm{~b}$ & $0,28-0,33$ \\
C20:1 & $\mathrm{E}$ & $0,35 \pm 0,02 \mathrm{a}$ & $0,31-0,37$ \\
Behénico & $\mathrm{A}$ & $0,12 \pm 0,01 \mathrm{a}$ & $0,09-0,14$ \\
C22:0 & $\mathrm{E}$ & $0,11 \pm 0,01 \mathrm{~b}$ & $0,09-0,13$ \\
Lignocérico & $\mathrm{A}$ & $0,03 \pm 0,00 \mathrm{~b}$ & $0,05-0,05$ \\
C:24:0 & $\mathrm{E}$ & $0,05 \pm 0,01 \mathrm{a}$ & $0,05-0,07$ \\
\hline Paracada a c & &
\end{tabular}

* Para cada ácido graso analizado medias seguidas de la misma letra no son significativamente diferentes $(P<0,05)$.

significativa se repite cada uno de los años considerados.

Los resultados más consistentes se obtuvieron para los ácidos C18:0, C18:3, C20:0 y C20:1, y para la susceptibilidad oxidativa; en todos ellos también resultaron significativas las diferencias entre variedades cada uno de los años del ensayo.

\subsection{Esteroles, ceras y alcoholes alifáticos}

Las dos variedades tienen un perfil de esteroles claramente diferente, como se indica en la Tabla 5. No se han encontrado diferencias significativas en el contenido de colesterol, estigmasterol y eritrodiol de las dos variedades. En otros esteroles como el campesterol, campestanol y $\beta$-sitosterol los valores medios de las dos variedades son similares, pero las pequeñas diferencias existentes son significativas, por lo que Arbequina tiene más campesterol y $\beta$-sitosterol aparente que Empeltre, pero menos campestanol. El resto de esteroles analizados marcan más las diferencias entre las dos variedades. Arbequina quintuplica el contenido de 24-metilencolesterol y triplica el de $\Delta 5$ avenasterol respecto al de Empeltre. Por el contrario, esta variedad tiene mucho más $\Delta 7$ estigmastenol y $\Delta 7$ avenasterol que Arbequina.

El total de esteroles ha resultado siempre mayor en Arbequina que en Empeltre, pero la diferencia en los aceites de 2007 no llegó a ser significativa.
Se ha encontrado una influencia del año sobre los valores de algunos esteroles: campestanol, $\Delta 5$ avenasterol, $\Delta 7$ avenasterol tuvieron valor superior en 2007, mientras que los esteroles totales fueron mayores en 2006.

El contenido en ceras fue superior en Arbequina cada uno de los años y en el conjunto de los dos años. Además hubo diferencias entre los dos años siendo el contenido en ceras de 2007 un 20\% menor que en 2006.

Los alcoholes alifáticos también han sido más abundantes en Arbequina que en Empeltre, aunque las diferencias solo han sido significativas en el análisis conjunto de los años.

\subsection{Análisis Cluster}

Para este análisis se han utilizado las observaciones correspondientes a los caracteres más importantes: polifenoles, estabilidad, ácidos grasos saturados, monoinsaturados y poliinsaturados, susceptibilidad oxidativa, esteroles totales, metilcolesterol, campesterol, campestanol, delta5avenasterol, delta7avenasterol, delta7estigmastenol, ceras y alcoholes alifáticos.

En la Figura 2 se presenta el dendrograma de los distintos aceites, que aparecen identificados en el eje $X$, por un número de 3 cifras. La primera cifra corresponde a la variedad, 1 para Arbequina y 2 
Tabla 4

Valores medios con desviación estándar y rango de variación del total de ácidos grasos saturados, insaturados, monoinsaturados, poliinsaturados y de sus relaciones en aceites de Arbequina (A) y Empeltre (E)

\begin{tabular}{lccc}
\hline Parámetro & Variedad & Media \pm Desvest & Rango \\
\hline \multirow{2}{*}{ Saturados (S) } & $\mathrm{A}$ & $16,5 \pm 0,9 \mathrm{a}$ & $14,7-17,8$ \\
& $\mathrm{E}$ & $16,7 \pm 0,7 \mathrm{a}$ & $15,6-18,0$ \\
Insaturados (I) & $\mathrm{A}$ & $83,4 \pm 0,9 \mathrm{a}$ & $82,1-85,1$ \\
\multirow{3}{*}{ Monoinsaturados(M) } & $\mathrm{E}$ & $83,2 \pm 0,7 \mathrm{a}$ & $82,1-84,3$ \\
& $\mathrm{E}$ & $71,3 \pm 2,8 \mathrm{a}$ & $67,4-76,1$ \\
Poliinsaturados(P) & $\mathrm{A}$ & $68,5 \pm 1,5 \mathrm{~b}$ & $65,9-70,5$ \\
& $\mathrm{E}$ & $12,2 \pm 1,9 \mathrm{~b}$ & $9,0-14,7$ \\
I/S & $\mathrm{A}$ & $14,8 \pm 1,3 \mathrm{a}$ & $13,3-17,1$ \\
& $\mathrm{E}$ & $5,1 \pm 0,4 \mathrm{a}$ & $4,6-5,8$ \\
M/P & $\mathrm{A}$ & $5,0 \pm 0,3 \mathrm{a}$ & $4,6-5,4$ \\
& $\mathrm{E}$ & $6,0 \pm 1,2 \mathrm{a}$ & $4,6-8,4$ \\
Oleico/linoleico & $\mathrm{A}$ & $4,7 \pm 0,5 \mathrm{~b}$ & $3,8-5,2$ \\
& $\mathrm{E}$ & $6,2 \pm 1,3 \mathrm{a}$ & $4,6-8,8$ \\
Suscept. Oxidativa & $\mathrm{A}$ & $4,8 \pm 0,5 \mathrm{~b}$ & $3,9-5,4$ \\
& $\mathrm{E}$ & $652 \pm 83 \mathrm{~b}$ & $517-766$ \\
& & $779 \pm 58 \mathrm{a}$ & $716-888$ \\
\hline
\end{tabular}

* Para cada carácter analizado medias seguidas de la misma letra no son significativamente diferentes $(P<0,05)$.

para Empeltre, y la segunda cifra corresponde al año. Como puede verse hay dos grupos diferentes que corresponden a las dos variedades. A su vez, dentro de cada grupo, las muestras de cada año forman subgrupos. No aparecen las muestras de Empeltre 2004 ya que el programa solo utiliza las observaciones con datos en todas las variables y ese año no se analizaron los esteroles de esta variedad, por ser su primera cosecha.

\section{CONCLUSIONES}

El estudio muestra que los aceites de Arbequina y Empeltre tienen diferencias en su composición, aun siendo cultivadas en las mismas condiciones agronómicas y ambientales. Por otra parte, hay una influencia de las condiciones meteorológicas en la composición de los aceites de las dos variedades, por lo que se encontraron diferencias entre los tres años.

\section{Dendrograma}

Método Ward. Distancia Euclídea

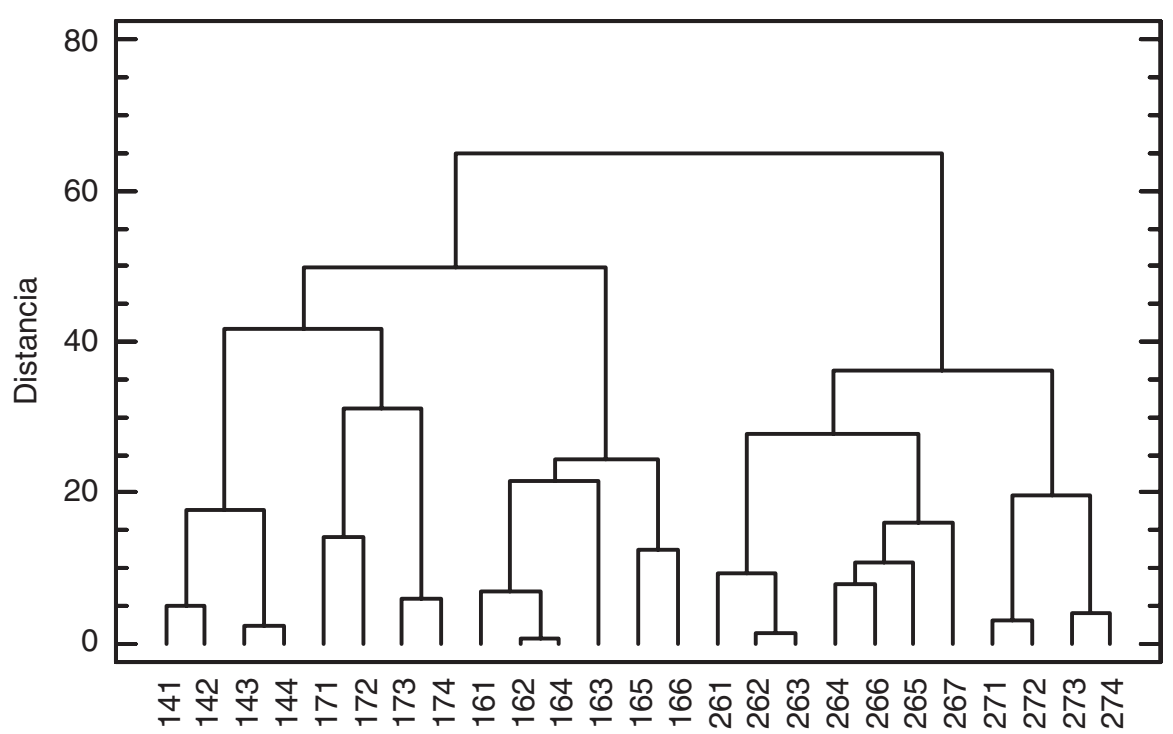

Figura 2

Dendrograma de los aceites de Arbequina y Empeltre, que aparecen identificados en el eje $\mathrm{X}$, por un número de tres cifras. La primera cifra corresponde a la variedad, 1 para Arbequina y 2 para Empeltre, y la segunda cifra corresponde al año. 
Tabla 5

Valores medios con desviación estándar y rango de variación de los esteroles, ceras y alcoholes alifáticos en aceites de Arbequina (A) y Empeltre (E)

\begin{tabular}{|c|c|c|c|}
\hline Parámetro & Variedad & Media \pm Desvest. & Rango \\
\hline Colesterol & $\begin{array}{l}A \\
E\end{array}$ & $\begin{array}{l}0,12 \pm 0,04 a \\
0,11 \pm 0,03 a\end{array}$ & $\begin{array}{l}0,10-0,20 \\
0,10-0,20\end{array}$ \\
\hline 24-Metilencolesterol & $\begin{array}{l}A \\
E\end{array}$ & $\begin{array}{l}0,93 \pm 0,13 a \\
0,18 \pm 0,06 b\end{array}$ & $\begin{array}{l}0,80-1,25 \\
0,10-0,30\end{array}$ \\
\hline Campesterol & $\begin{array}{l}A \\
E\end{array}$ & $\begin{array}{l}3,37 \pm 0,22 a \\
2,96 \pm 0,17 b\end{array}$ & $\begin{array}{l}3,00-3,70 \\
2,50-3,20\end{array}$ \\
\hline Campestanol & $\begin{array}{l}A \\
E\end{array}$ & $\begin{array}{l}0,40 \pm 0,08 b \\
0,48 \pm 0,08 a\end{array}$ & $\begin{array}{l}0,30-0,50 \\
0,40-0,60\end{array}$ \\
\hline Estigmasterol & $\begin{array}{l}A \\
E\end{array}$ & $\begin{array}{l}0,82 \pm 0,10 a \\
0,92 \pm 0,35 a\end{array}$ & $\begin{array}{l}0,70-1,00 \\
0,60-1,50\end{array}$ \\
\hline$\beta$-Sitosterol aparente & $\begin{array}{l}A \\
E\end{array}$ & $\begin{array}{l}95,2 \pm 0,20 a \\
94,1 \pm 0,38 b\end{array}$ & $\begin{array}{l}94,8-95,4 \\
93,6-94,8\end{array}$ \\
\hline$\Delta-5$ Avenasterol & $\begin{array}{l}A \\
E\end{array}$ & $\begin{array}{c}23,2 \pm 1,7 \mathrm{a} \\
7,0 \pm 1,4 \mathrm{~b}\end{array}$ & $\begin{array}{c}19,5-25,2 \\
5,0-9,3\end{array}$ \\
\hline$\Delta-7$ Estigmastenol & $\begin{array}{l}A \\
E\end{array}$ & $\begin{array}{l}0,15 \pm 0,04 b \\
0,62 \pm 0,14 a\end{array}$ & $\begin{array}{l}0,10-0,20 \\
0,50-0,90\end{array}$ \\
\hline$\Delta-7$ Avenasterol & $\begin{array}{l}A \\
E\end{array}$ & $\begin{array}{l}0,38 \pm 0,09 \mathrm{~b} \\
1,24 \pm 0,31 \mathrm{a}\end{array}$ & $\begin{array}{l}0,20-0,50 \\
0,90-1,90\end{array}$ \\
\hline Eritrodiol + uvaol & $\begin{array}{l}A \\
E\end{array}$ & $\begin{array}{l}0,99 \pm 0,38 b \\
1,43 \pm 0,48 a\end{array}$ & $\begin{array}{l}0,60-1,80 \\
0,90-2,10\end{array}$ \\
\hline Esteroles totales & $\begin{array}{l}A \\
E\end{array}$ & $\begin{array}{c}1843 \pm 257 a \\
1666 \pm 81 b\end{array}$ & $\begin{array}{l}1495-2157 \\
1569-1815\end{array}$ \\
\hline Ceras & $\begin{array}{l}A \\
E\end{array}$ & $\begin{array}{l}132 \pm 18 a \\
108 \pm 25 b\end{array}$ & $\begin{array}{c}105-161 \\
79-148\end{array}$ \\
\hline Alifáticos & $\begin{array}{l}A \\
E\end{array}$ & $\begin{array}{l}123 \pm 29 a \\
100 \pm 14 b\end{array}$ & $\begin{array}{l}74-174 \\
79-123\end{array}$ \\
\hline
\end{tabular}

Las diferencias más notables se encontraron en el índice de peróxidos y polifenoles totales con valores muy superiores en la variedad Empeltre, no obstante esta variedad presenta una mayor susceptibilidad oxidativa en todos los casos. Excepto dos muestras de Empeltre correspondientes al año 2007 en las que el índice de peróxidos superó el máximo admisible de 20 meq de $\mathrm{O}_{2} / \mathrm{kg}$ de aceite todos los demás pudieron clasificarse como virgen extra.

Los valores de los distintos parámetros analizados se asemejan notablemente a los obtenidos en otros trabajos sobre estas dos variedades, con las diferencias atribuibles a la edad de los árboles y a las condiciones de cultivo.

\section{AGRADECIMIENTOS}

Este trabajo fue financiado por INIA (Proyectos RTA01-001 y RTA04-050). Agradecemos la ayuda prestada por el personal de la Unidad de Suelos y Riegos del CITA en los trabajos de campo y por el de la Unidad de Grasas del Laboratorio Agroalimentario, en los análisis de los aceites.

\section{REFERENCIAS}

Allouche Y, Jiménez A, Gaforio JJ, Uceda M, Beltrán G. 2007. How heating affects extra virgin olive oil quality indexes and chemical composition. J. Agric. Food. Chem. 55, 9646-9654.

Aparicio R, Roda L, Albi M.A, Gutiérrez F. 1999. Effect of various compounds on virgin olive oil stability measured by Rancimat. J. Agric. Food Chem. 47, 4150-4155.

Cert A, Alba J, León-Camacho M, Moreda W, Pérez-Camino MC. 1996. Effects of talc adition and operating mode on the quality and oxidative stability of virgen oils obtained by centrifugation. J. Agric. Food. Chem. 44, 3930-3934.

Faci $\mathrm{JM}^{\mathrm{a}}$, Berenguer Maㅡ, Espada JL, Gracia, S, 2002: Effect of Variable Water Irrigation Supply in Olive (Olea Europaea L.) cv. Arbequina in Aragon (Spain). II. Extra Virgin Oil Quality Parameters. ACTA HORTICULTURAE, Proc.Fourth Int. Symposium on Olive Growing, 586, 649-652, Leuven (Bélgica).

Fereres E, Pruitt WO, Beutel JA, Henderson DW, Holzapfel E, Shulbach H, Uriu K. 1981. ET and drip irrigation scheduling. En Fereres E. (Ed.) Drip irrigation management. University of Califirnia. Div. of Agric. Sci. № 21259 págs. 8-13.

García-Fernández MD, Berengena J. 1993. Respuesta del olivo a diferentes dosis de agua de riego. Estimación de coeficientes de cultivo. XI Jornadas Técnicas de Riegos. Valladolid. Págs. 107-113.

Gracia S. 1996. El olivar y su aceite en Aragón. Oleo (Especial SIO-96), 44-46.

Gracia Ma S. 2001. Composición química de distintas calidades de aceite de oliva virgen de la variedad "Empeltre" en el Bajo Aragón. Grasas y Aceites 52: 52-58. 
Gracia M ${ }^{a}$ S, Marco P, Bielsa S, Espada JL Arranz E. 2005. Estudio del momento óptimo de la recolección en la variedad "Empeltre , en el Bajo Aragón. XII Simposium científico-técnico Expoliva. Foro de la Tecnología oleícola y la calidad. TEC-30. Jaen 12-13 mayo 2005.

Gutiérrez F. 1989. Determinación de la estabilidad oxidative de aceites de oliva virgenes: comparación entre el Método del oxígeno activo (A.O.M.) y el método Rancimat. Grasas y Aceites 40,1-5.

Mailer R, Conlan D, Ayton J. 2005. Olive Harvest: Harvest Timing for Optimal Olive Oil Quality. A Report for the Rural Industries Research and Development Corporation. RIRDC Project No DAN 179A.

M.A.P y A. 2007. Anuarios de Estadística Agraria. Ministerio de Agricultura, Pesca y Alimentación, Madrid.

Poiana M, Mincione A. 2004. Fatty acids evolution and composition of olive oils extracted from different olive cultivars grown in Calabrian area. Grasas y Aceites 55, 282-290.

Reglamento CEE №2568/91 de la Comisión del 11 de julio de 1991, relativo a las características de los aceites de oliva y de los aceites de orujo de oliva y sobre sus métodos de análisis. DOCE № L248. Anexos II y III.

Reglamento CEE 183/93 del 16 de febrero de 1993.
Reglamento no 796/2002 de 6 de mayo de 2002.

Salas J, Pastor M, Castro J, Vega V. 1997. Influencia del riego sobre la composición y características organolépticas del aceite de oliva. Grasas y Aceites 48, 74-82.

Salvador MD, Aranda F, Fregapane G. 2001. Influence of fruit ripening on "Cornicabra virgin olive oil quality. A study of four successive crop seasons. Food Chemistry 73, 45-53.

SAS versión 9.1.SAS Institute Inc., Cary, NC. USA.

Torres MM., Maestri DM. 2006. Chemical composition of Arbequina virgen olive oil in relation to extraction and storage conditions. J. Sci. Food Agric. 86, 2311-2317.

Tous J, Romero A, Plana J, Guerrero L, Díaz I, Hermoso F. 1997. Características de los aceites de oliva virgen de la variedad Arbequina. Fruticultura Profesional. Especial Olivicultura, 11, 118-124.

Tous J, Romero A, Plana J, Espada JL, Gracia M른, Lizar B, Rallo J, Martorell A, Iñiguez A, García J, Elguea JA. 2004. Ficha varietal del cultivar "Empeltre". Olivae 102, 31-32.

Vázquez, A, Janer del Valle C, Janer del Valle M. 1973. Determinación de polifenoles totales del aceite de oliva. Grasas y Aceites, 24, 350-357.

Recibido: 22/10/08 Aceptado: 18/11/08 